\title{
EL CONCEPTO DE “CLUB” EN ORTEGA Y HUIZINGA
}

\author{
TARO TOYOHIRA \\ bttps://orcid.org/0000-0002-6719-7253 \\ Universidad de Salamanca \\ bttp://dx.doi.org/10.15304/ag.39.2.6343
}

\section{Resumen}

El presente estudio investiga la influencia de José Ortega y Gasset sobre la teoría del juego de Johan Huizinga. Analiza especialmente la distinción entre el juego auténtico y el pueril (Huizinga), el deporte y el pasatiempo (Ortega) y el concepto de club en ambos autores. El club es una comunidad originaria por la cual el espíritu lúdico entra e interviene en el mundo cotidiano. Según ambos autores, el espíritu lúdico deportivo crea el Estado y todas las instituciones culturales políticas a través de este extraño tipo de comunidad.

Palabras clave: Ortega y Gasset, Huizinga, juego, deporte, club.

\section{Abstract}

The present study investigates the influence of José Ortega y Gasset on the play theory of Johan Huizinga. It especially analyzes the distinction between authentic and childish play (Huizinga), sport and pastime (Ortega) and the concept of club in both authors. The club is an original community through which the playful spirit enters and intervenes in the mundane world. According to both authors, the sports spirit creates the State and all political cultural institutions through this strange type of community.

Keywords: Ortega y Gasset, Huizinga, play, sport, club.

Recibido: 15/10/2019. Aceptado: 18/02/2020. 


\section{Introducción}

Es muy conocido el contacto personal entre el filósofo español José Ortega y Gasset y el historiador holandés Johan Huizinga. El propio Ortega testimonia que "en conversaciones privadas Huizinga me expresó muchas veces en qué medida le habían movido a emprender su gran obra [Homo ludens] las breves insinuaciones hechas por mí sobre el tema" ". Por su parte Huizinga invita al filósofo español para dar conferencias en los Países Bajos en 1936 y $1937^{2}$. No obstante, su relación teórica no es demasiado estudiada. Incluso a veces se niega la influencia de Ortega sobre Huizinga, a pesar del testimonio orteguiano ${ }^{3}$. El presente estudio se opone a esta postura y sostiene que Ortega tuvo una fuerte influencia sobre uno de los conceptos centrales en la teoría del juego de Huizinga: el "club". Si bien, en un principio, este concepto puede parecer marginal, en realidad, posee una importancia decisiva en la teoría de ambos pensadores.

\section{El juego y el deporte}

En primer lugar, el concepto de "club" tiene un sentido muy peculiar en Ortega y Huizinga. Por ejemplo, no se debe entender por este término, el "club" de fútbol o el de baloncesto, etc., como Real Madrid o F.C. Barcelona. Como señala el propio Huizinga, el deporte en la sociedad moderna ya ha perdido su esencia lúdica y se acerca cada vez más a una actividad "seria", que es el negocio, sin convertirse en él de todo: "La actitud del jugador profesional ya no es la verdadera actitud de juego: dejan de existir para él la espontaneidad y la despreocupación. En la sociedad moderna, el deporte se aleja cada vez más de la esfera del juego puro, para convertirse en un elemento sui generis; deja de ser juego, pero no llega a ser seriedad"4. Aunque el deporte nunca "llega a ser seriedad" completamente (puesto que, al fin y al cabo, siempre tendrá algo de juego) el "club" de la sociedad moderna

${ }^{1}$ J. Ortega y Gasset, “Anejo I. Máscaras” (1946), IX, p. 866. Las referencias son de la edición de Obras completas, 10 vols. Madrid. Fundación José Ortega y Gasset / Taurus. Señalamos el número de tomo en romanos y de página en arábigos.

2 J. Gracia, José Ortega y Gasset, Madrid, Taurus, 2014, p. 487.

3 "Como recordará con gusto alguna otra vez, le había confesado en privado 'muchas veces en qué medida le habían movido a emprender su gran obra las breves insinuaciones hechas por mí sobre este tema', aunque no hay rastro de Ortega en el libro de Huizinga". Ibid., p. 573.

${ }^{4}$ J. Huizinga, Homo ludens, Lisboa, Azar, 1944, p. 253. 
es casi una empresa comercial y "seria" que se aprovecha del juego; y los deportistas son sus empleados contratados. Por su parte, Ortega también indicaba que el deporte de hoy en día ya había perdido la autenticidad y se convirtió en una "manía". Es decir, ocupa un puesto demasiado importante en la sociedad y ya no es una actividad "lúdica" y "superflua": "Está bien alguna dosis de fútbol. Pero ya tanto es intolerable. Y lo mismo digo de los demás deportes físicos. La prueba está en los periódicos, que por su naturaleza misma son el lugar donde más pronto y más claramente se manifiesta lo falso de cada época. [...] Son ya demasiadas las columnas y las páginas que dedican a los ejercicios corporales" ${ }^{6}$. Es curioso que los dos pensadores que reivindicaron más entusiastamente la importancia del juego y el deporte coincidieran en negar el auténtico espíritu lúdico deportivo al hombre moderno. Esto se debe al hecho de que, tanto para uno como para el otro, el verdadero espíritu lúdico deportivo - es decir, ya no se trata del juego o del deporte en sí mismos sino del espíritu que hay detrás de ellosconsista en un difícil equilibrio entre los aspectos contradictorios de la "seriedad" y la "superfluidad", el "placer" y el "ascetismo", la "libertad" y la "obediencia". Cuando el juego o el deporte pierden este difícil equilibrio, se convierten en un mero "pasatiempo" o un "juego pueril" por eso ambos distinguen entre el "juego" y el "deporte” (Ortega), el "juego auténtico" y el "juego pueril” (Huizinga):

5 “Todo, desde la manía del deporte físico (la manía, no el deporte mismo) hasta la violencia en política; desde el 'arte nuevo' hasta los baños de sol en las ridículas playas a la moda. Nada de eso tiene raíces, porque todo ello es pura invención, en el mal sentido de la palabra, que la hace equivaler a capricho liviano. No es creación desde el fondo sustancial de la vida; no es afán ni menester auténtico. En suma: todo eso es vitalmente falso". J. Ortega y Gasset, La rebelión de las masas (1930), IV, p. 492.

6 J. Ortega y Gasset, "Revés del almanaque” (1930), II, pp. 817.

${ }^{7}$ Para Huizinga, cuando el juego pierde su espontaneidad y la despreocupación se convierte en un "juego pueril". Precisamente por eso, el deporte de hoy en día es una manifestación del puerilismo de la sociedad contemporánea: "El niño que juega no es pueril. Sólo llega a serlo cuando el juego empieza a aburrirle o no sabe a qué jugar. Si el puerilismo general de hoy en día fuese un juego auténtico, habría que ver en ello un signo de que la sociedad vuelve hacia las formas culturales arcaicas en que el juego era un viviente factor productivo. Muchos estarán inclinados acaso a considerar el progresivo 'reclutamiento' de la sociedad, efectivamente, como una primera etapa de tal retorno. A nuestro juicio, están equivocados. Sólo logramos reconocer un signo amenazador de disolución en todos los fenómenos de un espíritu que renuncia voluntariamente a su madurez. Faltan las características esenciales del juego verdadero, aun cuando el comportamiento pueril adopta muchas veces, exteriormente, la forma del juego. La cultura tendrá que seguir otro sendero para recuperar la dignidad, la consagración y el estilo”. J. Huizinga, Homo ludens, ed. cit., pp. 263-264. 
En efecto, el estado de espíritu que a la Teoría corresponde no es el de la última y pavorosa seriedad de la vida, no es la emoción religiosa sino la alciónica jovialidad del deporte. [...] Estoy evitando emplear la palabra juego que, por un lado, les aclararía la cuestión pero no lo he hecho porque la aclaración sería esta vez excesiva, quiero decir, falsa. [...] El juego es irresponsable y no crea nada —es pasatiempo. El pensamiento, en cambio, crea concepciones del mundo y de la vida, que transustanciadas en creencias, serán como vastos continentes en los cuales vivirá la humanidad alojada, a veces, durante milenios. [...] Quedémonos por tanto en un justo medio entre la abrumadora seriedad del vivir y la irresponsable liviandad del jugar. Este punto medio es el deporte que tiene del vivir la dura disciplina, el enérgico esfuerzo, y tiene del jugar que ese esfuerzo no nos es impuesto sino engendrado por nuestro albedrío ${ }^{8}$.

Ortega no siempre sigue esta distinción entre el juego y el deporte. En ocasiones emplea los dos términos indistintamente, puesto que, al fin y al cabo, el deporte es una subcategoría de la categoría-juego. En Huizinga también se puede reconocer esta distinción entre el juego pueril y el juego auténtico. Para el pensador holandés el juego es algo espontáneo, libre y voluntario. En cuanto alguien le obliga a jugar al jugador, el juego deja de ser juego'. Sin embargo, dentro del juego mismo, el jugador no puede ser del todo libre. Debe respetar las reglas. En este sentido, el jugador se impone a sí mismo, libre y voluntariamente, unas reglas. Son reglas, hasta cierto grado, "sagradas", dado que el jugador no debe cuestionar sus fundamentos: "Paul Valéry dijo una vez al respecto - y es esto una idea de un alcance extraordinario- que en cuanto a las leyes del juego no hay escepticismo posible. Es que el fundamento en que se basan es inmovible. En cuanto se falta a las reglas, el mundo del juego se desploma" ${ }^{10}$, afirma Huizinga. Por eso, dice Ortega también, que "el juego es precisamente lo que no puede existir sin una peculiar seriedad que consiste en "cumplir sus reglas"”"11.

De este modo, para ambos, el "auténtico juego" (Huizinga) y el "deporte" (Ortega) muestran caracteres muy complejos. Por un lado, es una actividad placentera, no obstante, también requiere un ascetismo severo y una dedicación despreocupada. Es espontáneo y libre, pero también exige un máximo respeto por cumplir las reglas. Es "superfluo", sin embargo, para el sujeto lúdico deportivo, tiene un aspecto "serio" y, hasta cierto grado,

${ }^{8}$ J. Ortega y Gasset, La razón histórica [curso de 1940], IX, pp. 485-486. Cursivas mías. Sobre la filosofía como una actividad deportiva, véase: N. Orringer, "Aurora del filosofar como deporte en Ortega”, en M. Garrido, N. Orringer (coords.), El legado filosófico español e hispanoamericano del siglo XX, Madrid, Cátedra, 2009, pp. 233-248.

${ }^{9} \mathrm{~J}$. Huizinga, Homo ludens, ed. cit., p. 28.

${ }^{10}$ Ibid., p. 26.

${ }^{11}$ J. Ortega y Gasset, La idea de principio en Leibniz y la evolución de la teoría deductiva (1947), p. 1149. 
"sagrado". Debido a este carácter complejo, ambos critican el "puerilismo" del juego y el trabajo en la sociedad contemporánea. Por un lado, el juego se acerca cada vez más a una actividad "seria" e interesada (un negocio); por otro, las actividades "serias" (por ejemplo, la política) comienzan a perder la "seriedad" y las masas intentan convertir todo en juego y diversión ${ }^{12}$. En esta crítica de la sociedad contemporánea se puede encontrar mucha influencia de Ortega sobre Huizinga; de hecho, éste utiliza la distinción orteguiana del "hombre noble" y el "hombre-masa" en Entre las sombras del mañana ${ }^{13}$. La diferenciación entre el juego pueril y el auténtico, el pasatiempo y el deporte, es muy importante, ya que sólo el auténtico juego y el deporte crean una extraña comunidad fuera del mundo lúdico, que es el "club".

\section{El “club”, puente entre el mundo lúdico y el mundo cotidiano}

Como señala Huizinga, todo tipo de juego, sea pueril o auténtico, crea un mundo "encantado", separado del mundo cotidiano: "el juego no es la vida 'cotidiana'. Es un modo de aislarse a una esfera de actividad temporal con una orientación propia" ${ }^{14}$. Ortega también indica que "las reglas de un juego - y no hay juego sin reglas — crean un mundo que no existe. [...] Dios hizo el mundo, este mundo; bien, pero el hombre hizo el ajedrez —el ajedrez y todos los demás juegos. El hombre hizo, hace... el otro mundo, el verdaderamente otro, el que no existe, el mundo que es broma y farsa" ${ }^{15}$.

12 "Por ejemplo: la propensión a hacer ocupación central de la vida los juegos y los deportes". J. Ortega y Gasset, La rebelión de las masas (1930), IV, p. 436; "Lo que, en efecto, no tiene sentido es querer hacer de la vida toda puro divertimiento y distracción, porque entonces no tenemos de qué divertirnos, de qué distraernos". Idea del teatro. Una abreviatura (1946), IX, p. 847; J. Huizinga, Homo ludens, ed. cit., pp. 262-263.

${ }^{13} \mathrm{~J}$. Huizinga, Entre las sombras del mañana: diagnóstico de la enfermedad cultural de nuestro tiempo (trad. María de Meyere), Madrid, Revista de Occidente, 1951, p. 200.

${ }^{14} \mathrm{~J}$. Huizinga, Homo ludens, ed. cit., p. 23.

${ }^{15} \mathrm{~J}$. Ortega y Gasset, Idea del teatro. Una abreviatura (1946), IX, p. 847. Cursivas del autor. Ortega resalta esta creación de "otro mundo aislado y separado del real" especialmente en el arte como juego: "La forma más perfecta de la evasión al otro mundo son las bellas artes y si digo que son la forma más perfecta de juego evasivo no es por ningún convencional homenaje, no es porque yo sienta lo que hace muchos años llamé 'beatería cultural', [...] sino porque consiguen, en efecto, libertarnos de esta vida más eficazmente que ninguna otra cosa". Ibid., p. 848. Este aislamiento estético es un aspecto recurrente en Ortega y se nota la fuerte influencia de Theodor Lipps, como hace notar Taro Toyohira: "De ahí que tanto para Lipps como para Ortega, el mundo estético esté compuesto de objetos totalmente diferentes de los reales, cuya presencia es 'absoluta' e 'indubitada'; y que forme una esfera 
Sin embargo, Ortega resalta que el "deporte" como un juego auténtico no sólo crea un mundo temporal o una "sociedad" provisional, que incluso un simple saludo puede crear", sino también un "club" durable fuera del mundo lúdico-deportivo, en el mundo real-cotidiano. El juego de policías y ladrones crea una "sociedad que dura un instante" o un "mundo encantado" que dura mientras juegan los niños, incluso entre desconocidos, pero no crea un "club". Sólo la "apasionada seriedad", casi religiosa, del "deporte" crea un "club" durable y estable fuera del mundo lúdico. Ortega sostiene que el Estado mismo nace como un "club", una "sociedad secreta", de los jóvenes que se agrupan y entrenan para raptar a las mujeres de otras tribus ${ }^{17}$.

Ambos pensadores afirman que todo deporte o juego auténtico tiene un delicioso sabor del secreto. No es un mero juego o un pasatiempo fácil del que puede participar y disfrutar cualquier persona. No deja entrar a aquéllos que no respetan y cumplen las reglas del juego apasionadamente. El placer de cada juego es secreto y exclusivo de los jugadores que respetan y cumplen apasionadamente las reglas:

La vida en común inspira la idea de construir un albergue estable y capaz, que no sea la guarida transitoria o la simple pantalla contra el viento. Y así ocurre que la primera casa que el hombre edifica no es la casa de la familia aun inexistente, sino el casino de los jóvenes. En ella preparan sus expediciones, cumplen sus ritos; en ella se dedican al canto, a la bebida y al frenético banquete común. Es decir, que el "club" es, quiérase o no, más antiguo que el hogar doméstico, como el casino que la casa.

Está prohibido, so pena mortal, a los hombres maduros, mujeres y niños entrar en el casino varonil, que, por sus formas subsecuentes, llaman los etnólogos la "casa de los solteros". Todo es en ella, misterioso, secreto y tabú. Porque es un hecho sorprendente que estas primitivas asociaciones juveniles suelen tener el carácter de sociedades secretas, de férrea disciplina interna, donde se cultivan las destrezas vitales de la caza y la guerra con un severo entrenamiento ${ }^{18}$.

Huizinga también señala que este sabor del secreto o la exclusividad del trato tiene un poder mágico que crea un grupo permanente fuera del mundo lúdico, que es el "club":

'aislada' e 'independiente'. [...] Este aislamiento o 'hermetismo' de la obra de arte aparecerá repetidamente en las obras de Ortega como, por ejemplo, en 'Meditación del marco', Ideas sobre la novela o Idea del teatro". T. Toyohira, "Theodor Lipps y el concepto de estilo en la estética orteguiana”, Revista de Estudios Orteguianos 35 (2017), p. 174.

${ }^{16}$ J. Ortega y Gasset, “El origen deportivo del Estado, II” (1925), III, p. 779.

17 "Vemos, pues, que la primera sociedad humana, propiamente tal, es todo lo contrario que una reacción a necesidades impuestas. La primera sociedad es esta asociación de jóvenes para robar mujeres extrañas al grupo consanguíneo y dar cima a toda suerte de bárbaras hazañas”. J. Ortega y Gasset, "El origen deportivo del Estado” (1924), II, p. 714.

${ }^{18}$ Ibid., pp. 713-714. 
La sensación de tener trato exclusivo, de estar dedicado conjuntamente a algo importante, de diferenciarse en común de otros y de sustraerse a las normas generales, ejerce un efecto mágico que sobrepasa la duración del juego. El club es para el juego, lo que el sombrero para la cabeza. Se pecaría de demasiado simplista si de repente se declarara que lo que la etnología llama "phratrien", comunidades de ancianos o liga de hombres, eran comunidades de juego. No obstante, se comprobará una y otra vez la dificultad de aislar las compañías permanentes, principalmente de las culturas arcaicas con sus finalidades muy graves, solemnes y aún sagradas, nítidamente de la esfera lúdica ${ }^{19}$.

El concepto de "club" posee una importancia decisiva en la teoría de ambos, puesto que tanto Ortega como Huizinga reconocen el origen de todas las instituciones culturales, sociales y políticas en esta peculiar forma de comunidad, que no está formada ni por la sangre ni por el oficio o por ninguna razón "seria", sino por una afición "desinteresada" y "superflua". Para ambos, el juego y el deporte crean la sociedad y la cultura, intervienen en la vida cotidiana, mediante la formación del "club". Es la puerta por la que el espíritu lúdico deportivo entra en la sociedad; el gozne que une la esfera lúdica con la vida cotidiana. Aunque en este pasaje Huizinga no nombra a Ortega, aquí es indudable la influencia del filósofo español, ya que es él, quien sostiene que "phratrien" o "fratría ( $\varphi \rho \alpha \tau \rho i ́ \alpha)$ " significa "comunidades de deportistas" o "asociación de fiesta y guerra", en suma, el "club", en "El origen deportivo del Estado":

Lo cierto es que dondequiera que presenciamos la incorporación verdaderamente originaria de un organismo político, dondequiera que entrevemos el nacimiento de un Estado hallamos la presencia del "club" juvenil, que danza y combate. [...] Por lo que hace a Grecia, estas instituciones se llaman file, fratría, hetairía. Los helenistas entienden el sentido de estas palabras, pero no entendían hasta hace poco qué cosas eran las así designadas. File significa tribu, pero no como unidad de consanguíneos, sino como cuerpo organizado de guerreros. Fratría significa hermandad, y hetairía, compañía. Antes de que exista la polis, la ciudad con su Constitución, el pueblo griego se hallaba estructurado en esas otras formas. Ahora bien; la fratría o hermandad, que tiene entre los arios asiáticos su correspondencia en la sabha, no es más que la clase de edad de los jóvenes, organizada en asociación de fiesta y guerra. No se olvide que, como he dicho, primitivamente los jóvenes llaman padres a todos los hombres de la clase más provecta, y se llaman entre sí hermanos ${ }^{20}$.

El "club" se forma por la afición y el placer comunes, pero no sólo y exclusivamente por ellos. Es, como el juego mismo, también una entidad muy compleja y contradictoria. Dentro de él, se fomentan la férrea disciplina interna, el severo entrenamiento y la competitividad entre los jugadores,

${ }^{19}$ J. Huizinga, Homo ludens, ed. cit., p. 27.

${ }^{20}$ J. Ortega y Gasset, “El origen deportivo del Estado” (1924), II, p. 716. 
puesto que, para Ortega, todo juego auténtico debe conllevar ciertas dificultades, metas difíciles de conseguir. Y es aquí donde el juego adquiere otro carácter propio del "deporte". Por ejemplo, señala Ortega, para la "caza como deporte", la "escasez de piezas" es una condición esencial e imprescindible: "Las palabras 'escasez' y 'abundancia' del usual vocabulario venatorio tienen una significación meramente relativa que cuenta ya, como supuesto tácito, con no superabundar nunca la caza; por tanto, con que, en el sentido absoluto del vocablo, es siempre escasa" ${ }^{21}$. El cazador deportivo no busca cualquier animal como su objeto. Busca precisamente los animales más escasos en cada época, que sólo se pueden encontrar en zonas geográficas muy limitadas ${ }^{22}$. Por eso, "ya al comenzar la historia de la caza era tenida como su técnica básica la de detectar el animal; por tanto, que es consustancial a toda la faena la escasez de las piezas" ${ }^{23}$. Lo mismo con el "deporte" de "cazar" a las mujeres de otras tribus. Ellas son también piezas escasas y difíciles de conseguir, y precisamente por eso, son objetos privilegiados y atractivos. Los jóvenes se agrupan con este objetivo común, "deciden robar las mozas de hordas lejanas. Pero esto no es una empresa suave: las hordas no toleran impunemente la sustracción de sus mujeres" ${ }^{24}$. Para robar a las mujeres, tienen que combatir con los hombres de otra horda. Por eso, los cazadores de mujeres se entrenan mutuamente y se imponen una disciplina para conseguir la meta difícil de alcanzar:

Es decir, que la asociación política originaria es la sociedad secreta y que si sirve para el placer y la bebida es, al propio tiempo, el lugar donde se ejercita el primer ascetismo religioso y atlético. Recuérdese que la más exacta traducción del vocablo ascetismo es "ejercicio de entrenamiento", y los monjes no han hecho sino tomarlo del vocabulario deportivo usado por los atletas griegos. Askesis era el régimen de vida del atleta, llena de ejercicios y privaciones. De donde resulta que el casino de los jóvenes, primera casa y primer "club" placentero, es también el primer cuartel y el primer convento ${ }^{25}$.

Dado que la meta es difícil de conseguir, las piezas son siempre escasas, hay siempre entrenamiento, disciplina y ascetismo; pero también una

${ }^{21}$ J. Ortega y Gasset, "Prólogo a Veinte años de caza mayor, del Conde de Yebes" (1943), IV, p. 297.

${ }^{22}$ Ibid., p. 298.

23 "Desde la gran fiera avecindada en las estepas desérticas, hasta la becada puntiaguda de las aguas mansas, todas las especies que importan al cazador, sin más excepción, acaso, que el conejo - y éste es un animal semidoméstico-, son de una inestabilidad biológica extrema. Cualquiera circunstancia desregla su vitalidad y las extingue. Por eso su localización ha sido siempre muy circunscrita a condiciones precisas de clima, suelo y altitud”. Ibid., p. 296.

${ }^{24}$ J. Ortega y Gasset, “El origen deportivo del Estado” (1924), II, p. 713.

${ }^{25}$ Ibid., p. 714. 
competitividad entre los jugadores auténticos o deportistas. De este modo, la "guerra" con otra horda también reviste aspecto "festivo": "El traje de guerra es el mismo que el traje de fiesta: la máscara. Y fiesta, caza y guerra permanecieron mucho tiempo indiferenciadas: por eso, casi todas las danzas primitivas son la estilización de gestos venatorios o beligerantes" 26 . Fue un "match" entre dos "clubes atléticos". Y como indica Carlos Morillas González, en uno de los capítulos de Homo ludens Huzinga analiza detenidamente los aspectos lúdicos de la guerra ${ }^{27}$. Para Huizinga, esta competitividad "agonal" supone la verdadera función creadora de la cultura ${ }^{28}$. Cualquier aspecto de la cultura tiene un momento "agonal" y se desarrolla de la forma competitiva y lúdica ${ }^{29}$.

Tanto Ortega como Huizinga sostienen que toda la cultura humana se produce a partir del espíritu deportivo, superfluo y lujoso ${ }^{30}$. No obstante, en cuanto a su dimensión sociológica, si el mundo lúdico es, por su esen-

${ }^{26}$ Ibid.

27 "Diversas variantes inciden en ello y exponen la fecundidad cultural a que la guerra lleva: la precisión ritual del duelo y la cortesía que lo acompaña, la lucha noble como ideal de vida, la lealtad, la entrega, es decir, el 'juego limpio' que se mantiene fiel a las reglas”. C. Morillas González, "Huizinga-Caillois: variaciones sobre una visión antropológica del juego", Enrahonar 16 (1990), p. 18.

${ }^{28}$ Véase: J. Huizinga, Homo ludens, ed. cit., pp. 67-95.

29 "En el proceso de crecimiento de cada cultura, la función y estructura agonales alcanzan su forma más bella, ya en un periodo arcaico. En la medida en que el material cultural se torna más complejo, abigarrado y complicado y en que la técnica de la vida social y profesional, tanto del individuo como de la colectividad, se organizan más detalladamente, se desarrolla por encima de terreno primitivo de la cultura, poco a poco, una capa de ideas, sistemas, conceptos, dogmas y normas, conocimientos y costumbres, que parecen haber perdido todo su contacto con el juego. Paulatinamente la cultura se vuelve seria y ya no concede al juego sino un papel secundario. El periodo agonal ha pasado". Ibid., p. 94.

30 "La actividad original y primera de la vida es siempre espontánea, lujosa, de intención superflua, es libre expansión de una energía preexistente". J. Ortega y Gasset, "El origen deportivo del Estado" (1924), II, p. 707; "Todo lo que posee un valor superior ha sido fruto y emanación de esfuerzos deportivos y superfluos. Y no por mero azar sino, en virtud, de una ley esencial. El trabajo no crea propiamente: hemos visto que era una actividad de adaptación. Cuando una necesidad nos apremia nos limitamos a buscar en el arsenal de nuestras posibilidades el medio más adecuado para satisfacerla. Pero ese arsenal, ese repertorio tenía que hallarse formado previamente a la necesidad sobrevenida. Buscando una fórmula un poco rigorosa habríamos de decir que el papel de la utilidad no es crear sino, más bien producir una selección entre nuestras posibilidades inútiles". J. Ortega y Gasset, "El sentido deportivo de la vitalidad" (1924), VII, p. 833; "la cultura nace en forma lúdica, o sea: al principio, la cultura se practicaba en forma de juego. Aún las actividades encaminadas directamente a la satisfacción de necesidades vitales, como por ejemplo la caza, gustan adoptar, en la sociedad arcaica, la forma de juego". J. Huizinga, Homo ludens, ed. cit., p. 67. Cursivas del autor. 
cia, aislada y separada del mundo cotidiano, haría falta un elemento para intervenir en la dimensión mundana social. Este elemento que permite a la actividad lúdica deportiva entrar en la esfera cotidiana es el "club", que está constituida por el espíritu lúdico deportivo, pero, perdura más allá de la duración del juego y se establece fuera de la esfera lúdica. Ortega sostiene que "el 'club' de los jóvenes crea, en la Historia, las cosas siguientes: "La exogamia. La guerra. La organización autoritaria. La disciplina de entrenamiento o ascética. La Ley. La asociación cultural. El festival de danzas enmascaradas o Carnaval. La sociedad secreta. Y todo ello, junto e indiferenciado, la génesis histórica e irracional del Estado" ${ }^{31}$.

\section{Conclusión}

En este estudio hemos intentado mostrar la influencia de Ortega sobre Huizinga y su afinidad teórica, sobre todo, en un concepto muy concreto: el "club". Es una forma de comunidad muy peculiar que existe a caballo entre la esfera lúdica y el mundo cotidiano. El espíritu lúdico deportivo crea el Estado y todas las instituciones culturales políticas a través del "club". También hemos estudiado la distinción entre el "juego auténtico" y el "pueril" (Huizinga) y el "deporte" y el "pasatiempo" (Ortega). Esta diferenciación tiene una estrecha relación con la crítica al "puerilismo" de la sociedad moderna en ambos autores. Por tanto, sería interesante un análisis comparativo más profundo del último aspecto en Ortega y Huizinga que no hemos podido hacer en este artículo.

\section{Bibliografía}

Gracia, J. (2014). José Ortega y Gasset. Madrid: Taurus.

Huizinga, J. (1944). Homo ludens. Lisboa: Azar.

Huizinga, J. (1951). Entre las sombras del mañana: diagnóstico de la enfermedad cultural de nuestro tiempo (trad. María de Meyere). Madrid: Revista de Occidente.

Morillas González, C. (1990). "Huizinga-Caillois: variaciones sobre una visión antropológica del juego", Enrahonar 16, pp. 11-39. https://doi.org/10.5565/rev/enrahonar.749

${ }^{31}$ J. Ortega y Gasset, “El origen deportivo del Estado” (1924), II, p. 715. 
Orringer, N. (2009). "Aurora del filosofar como deporte en Ortega", en Garrido, M., Orringer, N. (coords.), El legado filosófico español e hispanoamericano del siglo XX. Madrid: Cátedra, pp. 233-248.

Ortega y Gasset, J. (2004-2010). Obras Completas, I-X. Madrid: Taurus / Fundación José Ortega y Gasset.

Toyohira, T. (2017). "Theodor Lipps y el concepto de estilo en la estética orteguiana”, Revista de Estudios Orteguianos, 35, pp. 161-187. 